\title{
Optimal cost-based strategies in mobile network offloading
}

\author{
Kimmo Berg \\ Department of Mathematics and Systems Analysis \\ Aalto University School of Science \\ Helsinki, Finland \\ kimmo.berg@aalto.fi
}

\author{
Michail Katsigiannis \\ Department of Communications and Networking \\ Aalto University School of Electrical Engineering \\ Helsinki, Finland \\ michail.katsigiannis@aalto.fi
}

\begin{abstract}
This article examines potential deployment strategies for mobile operators to satisfy the high anticipated data traffic volume in their mobile networks. The mobile network offloading is analyzed with the traditional evolution of macrocellular radio access networks. Cognitive radio is considered as a technology that may augment these deployment strategies. A duopoly model is developed to examine where and when mobile offloading is likely to happen. The results show how much offloading there will be and the exact conditions when it is profitable based on the estimated cost parameters and the future demands. An illustrative case study in Helsinki metropolitan area is examined for year 2015.
\end{abstract}

Index Terms-mobile offloading, capacity investment, duopoly market, cognitive radio

\section{INTRODUCTION}

The usage of smart terminals and laptops as well as the proliferation of mobile applications create an enormous amount of data traffic in the mobile networks. Ericsson [1] predicts that within the next five years (2011-2016), mobile data traffic will grow 10 times as the number of mobile broadband subscriptions increases from 900 million to almost 5 billion globally. Focusing on the Finnish market, the number of mobile broadband connections have outnumbered all the other broadband subscriptions in 2010 [2]. As a result, the mobile data volume has increased rapidly and a higher growth rate is expected due to the ambitious national broadband policy. As a continuation of the universal obligation of $1 \mathrm{Mbps}$ to all citizens by 2010, a broadband project was launched to connect nearly all permanent residences, offices of businesses and public administration bodies to Internet with $100 \mathrm{Mbps}$ data rate through a fixed or wireless subscriber line of no more than two kilometers' length linked to the network by 2015 [3]. Therefore, within the next few years, huge investments are needed in the mobile communications sector to fulfill the requirements of the national broadband plan.

However, there is uncertainty about the optimal deployment strategies for the evolution of the radio access networks under the high anticipated data traffic growth. The mobile operator's general set of strategies includes investments on macrocellular network in a traditional way and investments on innovative technologies such as mobile network offloading and cognitive radio. The purpose of this paper is to find the most profitable deployment strategy for a mobile operator based on the future mobile data volume and the cost parameters, and its effect on the other mobile operator's profits in a duopoly market. The contribution of this study is the modeling of mobile operators' utility functions for different deployment strategies, highlighting the demand level in which the optimal strategy changes for one mobile operator. The model is also applied to a real case study in Helsinki metropolitan area.

The remainder of this paper is outlined as follows. In Section II, two different deployment strategies are discussed for the potential radio access network evolution: traditional macro cellular networks and mobile network offloading. Cognitive radio systems are suggested to enhance these two strategies. Section III describes the duopoly model and presents the analytical results. In Section IV, we show numerical results for two mobile operators in Helsinki metropolitan area. Finally, conclusions are drawn in Section V.

\section{Radio Access Network Evolution}

\section{A. Macro Cellular Networks}

The tendency for mobile operators is to follow an evolutionary strategy path based on the transition to High Speed Packet Access (HSPA+) and Long Term Evolution (LTE) technologies by utilizing the remaining spectrum allocations. From a technical perspective, there are three generic ways to improve network capacity: mobile operators can try to find more spectrum for mobile broadband via auctions or spectrum refarming, use radio technologies with better spectrum efficiency, and/or deploy the wireless base stations more densely. The current spectrum allocations are still able to cover the short-term capacity requirements, as HSPA+ and LTE improve the spectrum efficiency of wide area networks via advanced radio techniques such as carrier aggregation, higher-order modulation and multiple-input and multiple-output (MIMO) technology. This traditional macrocellular deployment strategy with the installation of upgraded cellular sites more densely might meet temporarily the coverage, capacity and quality requirements. However, the physical limitations of spectrum resources and the spectrum efficiency beyond LTE restrict capacity growth per site for future needs. Thus, the future macrocellular network deployment requires large number of base stations which incur high investments and operating costs. Therefore, the provisioning of increasingly high-speed 
data services is likely to require a new network deployment strategy.

\section{B. Mobile Network Offloading}

In fact, people spend most of their time at home and the office, and the majority of smartphone usage takes place indoors [4]. Around $70-80 \%$ of the mobile data traffic is generated indoors [5] and this fraction can increase up to $95 \%$ by 2015 [6]. However, the radio signals incur significant additional attenuation when they are transmitted from outdoor environment to indoor due to the building penetration losses. The signal degradation results in reduced quality of service in indoor places, and subsequently macrocells need to dedicate more radio resources for indoor connections to compensate the degradation. This poses significant challenges to the mobile network operators in coping with the increasing capacity and coverage demands for their high-speed data network infrastructure.

Mobile network offloading as a deployment strategy, especially in densely populated areas such as metropolitan areas, might provide a good solution to mobile operators [7]. Mobile network offloading, also called as mobile data offloading, is the use of complementary network technologies to deliver mobile data traffic originally planned for transmission over cellular networks [8]. This article focuses on local area networks, i.e., the use of smaller cells covering local, indoor areas as a complementary network technology, which defines the socalled wide-to-local area offloading. The main technologies for wide-to-local area offloading are 3GPP femtocells and IEEE WiFi. Small cells or access points are low-cost, low-power wireless base stations that are designed to provide high quality wireless service. They can operate in licensed and unlicensed spectrum and utilize the user's existing broadband Internet access as a backhaul link. Moreover, cognitive radio oriented access points has gained popularity in research community. In the medium-to-long term, small cells are needed to provide more capacity and better coverage cost-efficiently. To some degree, also the outdoor-placed base station density can be increased by microcell and picocell solutions. However, the indoor deployment of access points is foreseen as a necessity in guaranteeing sufficient coverage, low latency, and improved power efficiency and battery life of terminal.

By serving users at homes, offices and public places through access points might have a positive impact on the operator's total cost. Less installation of wide area radio equipment is needed, which cause reduction in capital (CAPEX) and operational expenditures (OPEX). The potential energy savings can reduce further the OPEX via lower energy bills, since the access points consume less power than macrocells. Hence, the cost reduction can create larger profit margin for the mobile operator. The co-existence of access points and macro cellular network would be beneficial not only for mobile operators but also for the end-users. Firstly, the service pricing can be low and affordable to all end-users. Furthermore, the better coverage and capacity improve customer satisfaction which results in less churn rate. Mobile operators can offer new value-added services and applications to the end-users via access points which could increase the user's utility and eventually the average revenue per user (ARPU) for the mobile operator. Consequently, through suitable integration of mobile offloading and the wide area radio access network, access points could improve the capabilities of the radio access network as well as its power consumption and increase quality of user experience.

\section{Cognitive Radio}

Most of the spectrum that attracts the mobile operators has already been assigned, and the current license-based spectrum management policy has resulted in low spectrum utilization [9]. Thus, the increasing demand for mobile data services results in crowding the radio frequency spectrum. So, the cost of additional mobile network capacity under the allocated licensed bands will increase as the bands become more congested in the future. The mobile networks already consist of several different radio access technologies and the installation of more base stations will be the only way, when the offloading deployment is not an option to increase capacity. The cost of the network will increase significantly and subsequently, this might affect the price of mobile services as well. Therefore, cognitive radio systems [10] are a promising solution and different spectrum sharing policies have been suggested [11]. Cognitive radio aims to reduce spectrum congestion by sensing unused bandwidth in the existing communication standards and opportunistically maximizes the spectrum utilization for the end user, thus enhancing the network capacity. Cognitive radio can be applied also to offloading network deployment, where the indoor access points are able to search the radio channel and estimate which resources are free among the available ones in order to avoid interference. The infrastructure requires a management server allowing to collect performance measurements from the access points and to manage the configuration of the access points [12].

In capacity problems, mobile operators can act as secondary operators. The cognitive radio turns the mobile operator's network to a secondary network which can dynamically lend and reuse the licensed spectrum allocated to other operators or other wireless systems (primary operators), under the condition that no harmful interference is caused to the primary services [13]. Thus, the mobile operator can provide cognitive radio services that improve coverage and maximize the data delivery across the existing communication standards utilizing a single base station antenna system. The intelligent cognitive radio base stations have a knowledge about its environment and its communication needs, and it can adapt its wireless transmissions in an optimal manner. On the other hand, especially in the case of joint macro and offloading network, after wide deployments of indoor access points, the macro network can have redundant capacity for secondary users and the mobile operator, as a primary operator, can make money by trading the unused spectrum.

Cognitive technologies are capable of increasing spectrum efficiency and network capacity significantly and offering 
mobile broadband services at lower cost. Finally, note that Finland's legislation allows the use of cognitive radio systems in the TV bands between $470-790 \mathrm{MHz}$, like the recent development in Europe and the USA [14], [15].

\section{Duopoly Model}

In this section we formulate a duopoly model to evaluate under what conditions the mobile data offloading is profitable to the operators. We compare four strategies: 1) wait and no investment in capacity, 2) invest in offloading, 3) invest in macrocells and 4) invest both in offloading and macrocells. The optimal strategy is derived as a function of future demands and the cost parameters.

\section{A. Model Description}

We consider a business case for two mobile operators (hereafter firms) in Helsinki metropolitan area with around one million inhabitants and with an area of 770 square kilometers. The mobile broadband subscriptions for each firm is around 400.000 with average total user data traffic of $2.9 \mathrm{~GB} / \mathrm{mon}$ for 2015. Helsinki metropolitan area is characterized by lower density of inhabitants than a typical western city, but the penetration rate of mobile broadband subscriptions is higher. We assume for simplicity that the demand does not depend on the firm's strategy, i.e., in reality the network may affect the quality of service and thus also the demand.

The total demanded traffic (in terabytes per month) for each firm is decomposed to downlink and uplink traffic with uplinkto-downlink ratio of $10 \%$. Furthermore, for the investigation of offloading strategy, we assume that the indoor downlink traffic is the main part with around $90 \%$ of the total downlink traffic. However, the maximum indoor traffic that can be satisfied by offloading is limited by the penetration of offloading service at the venues (households, offices, public places) in which the indoor access points are installed. The usage-based revenues (in million euro/year) are assumed to be constant per one $\mathrm{TB} / \mathrm{mon}$ and the revenues do not depend on the strategy. Again, we note that the different strategies may affect how much revenues the network generates.

As a reference point for the current total network capacity and the total cost of the network, the two firms operate only HSDPA (High Speed Data Packet Access) network, offering mobile broadband service. The maximum theoretical capacity for the cells is 3.6 Mbps and 14.4 Mbps in 2100 and $900 \mathrm{MHz}$, correspondingly. The model examines what is the optimal strategy as the mobile data traffic increase above the current total network capacity.

The strategy to invest in macro has two options. The first is to expand network capacity with the installation of new sites using the same HSDPA technology. The second is the replacement of the existing sites with HSPA+ (Evolved HSDPA). The maximum theoretical capacity for the upgraded cell is $42 \mathrm{Mbps}$ which replaces the HSDPA at $900 \mathrm{MHz}$ and $84 \mathrm{Mbps}$ which replaces HSDPA at $2100 \mathrm{MHz}$. The fixed cost for macro consists of the discounted depreciations of the previous network, the cost for the lease of the transmission lines, the site lease, the salary of network related personnel and the yearly decomposed cost of the license fee. The variable cost is the extra transmission lines and new network elements costs which are needed to "produce" more capacity for the network. Additionally, for the case of invest in macro with new site installation, the site lease is a variable cost [16], [17]. The cost comparison of the two different options reveals that the option to expand capacity by upgrading the base stations has lower marginal cost making it preferable when the demand is high. This is the case where a new technology might have higher fixed cost but the marginal cost per produced unit keeps the total cost at low level.

The strategy to invest in offloading, i.e., the installation of indoor access points, requires new investment from the scratch. The fixed cost for offloading is the depreciations of the cost for required network elements (Home NodeB gateways and the close subscription group list server) for the preparation of the firm to launch commercially the offload service. While the penetration rate increases and therefore the indoor downlink demanded traffic, the variable cost is the price of the access points, which are given to the venue owner, and the expansion of the offloading related core network elements [18], [19]. We assume that the variable costs are lower in business and public areas where the user density is higher than in homes. Thus, we have increasing variable cost as a function of capacity, which means that the access points are first installed in most costeffective places and the costs increase as these hot spots are covered.

\section{B. Mathematical Model}

Let us now construct the duopoly model. The operators are denoted by $i \in I=1,2$. The firms' current capacities are $k_{i}$, and it is assumed that the future demands $d_{i}, i \in I$, are known. The required capacity to be built is then $r_{i}=d_{i}-k_{i}$. The utility of a firm is the revenue generated by its customers minus the capacity costs. The utility from scenario 1 (no investment) is normalized to zero, and the revenue from the extra demand per capacity unit is assumed to be constant $a_{i}$. We note that the demands and the revenues could depend on the strategies since the investments in capacity affect the quality of service.

The capacity cost of macrocells is assumed to be linear

$$
c_{i}^{m}\left(r_{i}\right)=f_{i}^{m}+m_{i}^{m} r_{i},
$$

where $f_{i}^{m}$ is the macro fixed cost and $m_{i}^{m}$ is the macro marginal cost. The capacity cost of offloading is quadratic

$$
c_{i}^{o}\left(r_{i}\right)=f_{i}^{o}+m_{i}^{o} r_{i}+q_{i}^{o} r_{i}^{2},
$$

where $f_{i}^{o}$ is the offloading fixed cost, $m_{i}^{o}$ is the marginal cost at zero, and $q_{i}^{o}$ is the rate of marginal cost increase. The maximum capacity that can be satisfied with offloading is $k_{i}^{*}$, which depends on the amount of indoor data traffic since only indoor traffic can be served with offloading. When the firm invests in both, the cost is a combination of these two

$$
c_{i}^{b}\left(r_{i}\right)=\left\{\begin{array}{l}
f_{i}^{b}+m_{i}^{o} r_{i}+q_{i}^{o} r_{i}^{2}, \text { when } r_{i} \leq \bar{k}_{i}, \\
f_{i}^{b^{\prime}}+m_{i}^{m}\left(r_{i}-\bar{k}_{i}\right), \text { otherwise }
\end{array}\right.
$$


where $f_{i}^{b}$ is the fixed cost, $\bar{k}_{i}=\min \left(k_{i}^{*}, k_{i}^{\prime}\right), k_{i}^{\prime}$ is the capacity where the marginal costs of macro and offloading are the same, i.e., $k_{i}^{\prime}=\left(m_{i}^{m}-m_{i}^{o}\right) / 2 q_{i}^{o}$, and $f_{i}^{b^{\prime}}=f_{i}^{b}+m_{i}^{o} \bar{k}_{i}+q_{i}^{o} \bar{k}_{i}^{2}$. The cost functions are illustrated in Figure 1.

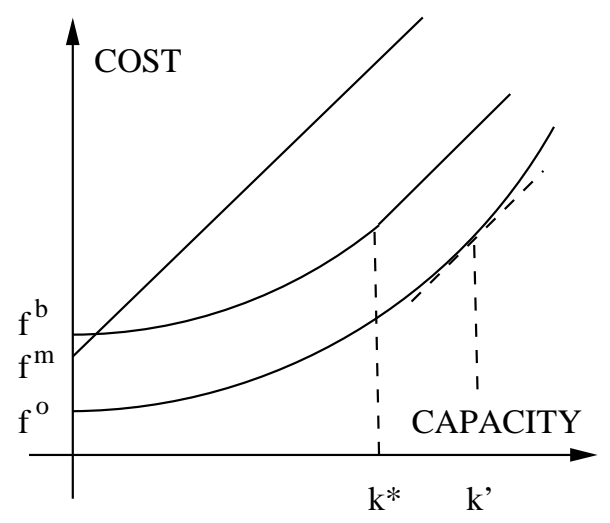

Fig. 1. The capacity costs in three scenarios.

With the wait and offloading strategies the firm may run out of capacity when the future demand is high. In this case the firm loses the exceeding demand to its competitor. The lost demand in scenario 1 is $l_{i}=r_{i}, i \in I$. When the firm offloads, the optimal capacity depends on the cost function. The value when the marginal cost equals the average revenue is $k_{i}^{\prime \prime}=\left(a_{i}-m_{i}^{o}\right) / 2 q_{i}^{o}$. When $k_{i}^{\prime \prime} \geq k_{i}^{*}$ then the lost demand in scenario 2 is $l_{i}=r_{i}-k_{i}^{*}$ and otherwise $l_{i}=r_{i}-k_{i}^{\prime \prime}, i \in I$. The total exceeding demands are then denoted by $r_{i}^{\prime}=r_{i}+l_{j}$, $i, j \in I$ and $j \neq i$. The firms' utilities with offloading are

$$
u_{i}\left(r_{i}^{\prime}\right)=\left\{\begin{array}{l}
a_{i} k-c_{i}^{o}(k), \text { if } r_{i}^{\prime} \geq k, \\
a_{i} r_{i}^{\prime}-c_{i}^{o}\left(r_{i}^{\prime}\right), \text { otherwise, }
\end{array}\right.
$$

where $k=\min \left(k_{i}^{*}, k_{i}^{\prime \prime}\right)$. With strategies 3 and 4 the utilities are

$$
u_{i}\left(r_{i}^{\prime}\right)=a_{i} r_{i}^{\prime}-c_{i}^{m / b}\left(r_{i}^{\prime}\right) .
$$

\section{Results}

In this section the player's subscript $i$ is omitted for simplicity. It is assumed that $m^{o}<m^{m}<a$ and $f^{b}>f^{m}>f^{o}$. Proposition 1 characterizes the optimal strategy for the firm as a function of the total future demand. We note that similar results could be derived when $f^{b}>f^{o}>f^{m}$.

First, let us find the values of demand when it is optimal to switch from the wait strategy 1 to some investment strategy 2 4. The values are solved from the equations when the utilities are at zero level and these are

$$
\begin{aligned}
& d_{12}=\left(a-m^{o}-\sqrt{\left(a-m^{o}\right)^{2}-4 q^{o} f^{o}}\right) / 2 q^{o}, \\
& d_{13}=f^{m} /\left(a-m^{m}\right), \\
& d_{14}^{a}=\left(a-m^{o}-\sqrt{\left(a-m^{o}\right)^{2}-4 q^{o} f^{b}}\right) / 2 q^{o}, \\
& d_{14}^{b}=\left(f^{b}+k^{*}\left(m^{o}-m^{m}+q^{o} k^{*}\right)\right) /\left(a-m^{m}\right) .
\end{aligned}
$$

When the utility from strategy 4 is positive at $k^{*}$, i.e., when $\left(a-m^{o}\right) k^{*} \geq f^{b}+q^{o}\left(k^{*}\right)^{2}$, it is optimal to switch from 1 to 4 at $d_{14}^{a}$ and otherwise at $d_{14}^{b}$.
Proposition 1: A) The optimal strategy is to wait when

$$
r^{\prime} \leq \min \left(d_{12}, d_{13}, d_{14}^{a} \text { or } d_{14}^{b}\right) .
$$

B) It is optimal to change from wait to strategy 2-4 when

$$
r^{\prime}=\min \left(d_{12}, d_{13}, d_{14}^{a} \text { or } d_{14}^{b}\right) .
$$

C) The value when it is optimal to change from 2 to 3 depends on the parameters. The value is

$$
d_{23}^{a}=\left(m^{m}-m^{o}+\sqrt{\left(m^{m}-m^{o}\right)^{2}+4 q^{o}\left(f^{m}-f^{o}\right)}\right) / 2 q^{o},
$$

if $d_{23}^{a} \leq \min \left(k^{*}, k^{\prime \prime}\right)$,

$$
d_{23}^{b}=\left(f^{m}-f^{o}+k^{*}\left(a-m^{o}-q^{o} k^{*}\right)\right) /\left(a-m^{m}\right),
$$

if $k^{*} \leq k^{\prime \prime}$ and otherwise

$$
d_{23}^{c}=\left(\left(a-m^{o}\right)^{2}+4 q^{o}\left(f^{m}-f^{o}\right)\right) /\left(4 q^{o}\left(a-m^{m}\right) .\right.
$$

D) For the high values of demand it is optimal to invest in both when the utility from 4 at $k=\min \left(k^{*}, k^{\prime \prime}\right)$ is higher than from 3 , i.e., if

$$
f^{m}-f^{b}+k\left(m^{m}-m^{o}\right)-q^{o}(k)^{2}>0 .
$$

If this holds then it is optimal to change from 2 to 4 at

$$
d_{24}^{a}=\left(\left(m^{m}-m^{o}\right) / 2+\sqrt{q^{o} \Delta}\right) / q^{o},
$$

if $d_{24}^{a} \leq \min \left(k^{*}, k^{\prime \prime}\right)$ and where $\Delta=f^{b}-f^{o}$,

$$
d_{24}^{b}=\left(\Delta+k^{*}\left(a-m^{m}\right)\right) /\left(a-m^{m}\right),
$$

if $k^{*} \leq \min \left(k^{\prime}, k^{\prime \prime}\right)$,

$$
d_{24}^{c}=\frac{4 q^{o}\left(k^{*}\left(a-m^{o}\right)+\Delta-q^{o}\left(k^{*}\right)^{2}\right)-\left(m^{m}-m^{o}\right)^{2}}{4 q^{o}\left(a-m^{m}\right)} .
$$

if $k^{\prime} \leq k^{*} \leq k^{\prime \prime}$, or

$d_{24}^{d}=\left(\left(a-m^{o}\right)^{2}-\left(m^{m}-m^{o}\right)^{2}+4 q^{o} \Delta\right) /\left(4 q^{o}\left(a-m^{m}\right)\right)$,

if $\max \left(k^{\prime}, k^{\prime \prime}\right) \leq k^{*}$.

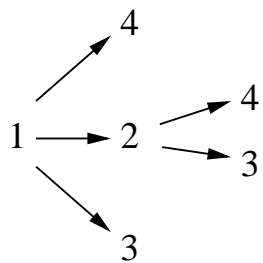

Fig. 2. The possible changes as the total demand increases.

The possible changes between the strategies are illustrated in Figure 2. When the demand increase is low, it is optimal to wait as Prop. 1A) suggests. When the demand increases, the value when to switch the strategy and to what investment strategy depends on the parameters, and the value is the minimum of $d_{12}, d_{13}$ and $d_{14}^{a}$ or $d_{14}^{b}$. For example, if the mimimum is $d_{13}$ then it is optimal to change to the macro investment at $r^{\prime}=d_{13}$. In this case, the macro investment remains optimal also for the higher demands. 
On the other hand, if the minimum is $d_{12}$ then it is optimal to switch to offloading at $r^{\prime}=d_{12}$. When the demand increases, it is optimal to switch to 4 if Eq. (8) holds, and otherwise it is optimal to switch to macro. There are two reasons to change from offloading to macro: the offloading capacity becomes limited and then the switching value is $d_{23}^{b}$, or the total cost of macro is lower. In this case the switching value is $d_{23}^{a}$ or $d_{23}^{c}$ depending on the parameters. Again, the macro investment remains optimal also for the higher demands. The same is true with strategy 4, i.e., if it is optimal to invest in both then this strategy remains optimal for the higher values of demand. This is due to the fact that the marginal costs are the same for strategies 3 and 4 when the demand is high, since they use the same technology.

The amount of offloading depends on the relation of $k^{*}$, $k^{\prime}$ and $k^{\prime \prime}$. In strategy 2 , it is optimal to offload up to the minimum of $k^{*}$ and $k^{\prime \prime}$, i.e., until the capacity becomes limited or the marginal cost equals average revenue per unit. The value $k^{\prime \prime}$ defines the limit when the density of indoor usage is high enough for the offloading to be profitable. For high density areas like large offices and public places the density is high enough but it may not be high enough for home residences with low usage. In strategy 4 , it is optimal to offload up to the minimum of $k^{*}$ and $k^{\prime}$. At $k^{\prime}$ when the marginal cost of offloading is equal to the marginal cost of macro then the firm should use macro for the higher demands. This means that the amount of offloading is constant for the high demands, and the access points are installed to locations that have high enough density for offloading to be more profitable than macro.

\section{CASe Study of Helsinki}

The parameters in the duopoly model are estimated to a case of Helsinki metropolitan area in 2015. We examine how the optimal strategies change as a function of firm 1's future demand. In this example, the optimal switching is 1-2-4 as the demand increases, i.e., from wait to offloading and finally to strategy 4 , where the offloading is used up to the value when the offloading and macro marginal cost are equal.

The cost parameters for offloading are $f_{i}^{o}=2, m_{i}^{o}=0.001$, $q_{i}^{o}=0.00003$, for macro $f_{1}^{m}=7, m_{1}^{m}=0.02, f_{2}^{m}=10$ and $m_{2}^{m}=0.016$, and for both $f_{i}^{b}=f_{i}^{o}+f_{i}^{m}, i \in I$. Firm 2 invests in more expensive macro technology so that the fixed cost is higher but the marginal cost is lower, since the new technology is more efficient. The average revenue per unit of capacity is $a_{i}=0.04, i \in I$. The exceeding demand for firm 2 is assumed to be $r_{2}=1000$.

The optimal strategies as a function of firm 1's demand $r_{1}$ are presented in Figure 3. The solid curves are for firm 1 and the dashed curves for firm 2. The optimal strategies 1-4 are shown in the lower right of the figure. We can see that the optimal strategy for firm 2 is always macro since the demand is so high and Eq. (8) does not hold, i.e., the macro strategy is more profitable than investing in both. The utility of firm 2 is not a constant but increases when firm 1 is capacity limited.

The optimal strategy for firm 1 is to wait when $r_{1} \leq d_{12} \approx$ 50 . When the demand is higher than this, the firm swithes

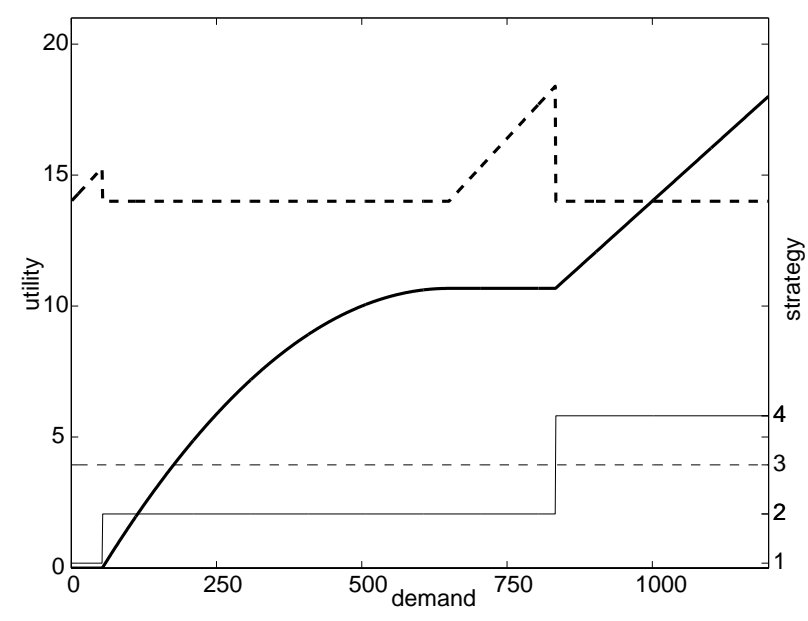

Fig. 3. The optimal strategies as a function of demand in the game.

to offloading. The utility increases fast with offloading as the marginal cost is low, and it almost reaches the utility of firm 2 even though the demand is about half of firm 2. At $r_{1}=$ $k_{1}^{\prime \prime} \approx 650$ the marginal cost reaches the marginal revenue and in this point the utility from offloading is at maximum. Thus, the firm does not build more capacity under offloading even though there is more demand. This extra demand goes to firm 2. When $r_{1}=d_{24}^{d} \approx 830$ the firm switches to strategy 4 and invests in both. The difference to the offloading strategy is that the firm has to pay for a higher fixed cost but gains lower marginal cost from the macro investment.

How much offloading is there? When the optimal strategy is offloading then all the new capacity is from offloading. But when the strategy is to invest in both then the amount of offloading is $k_{1}^{\prime} \approx 320$, since $k_{1}^{\prime} \leq k_{1}^{*}=900$. The value $k_{1}^{\prime}$ is the limit when the marginal costs are equal. Thus, when $r_{1}>830$ there is less offloading than if $320 \leq r_{1}<830$.

\section{CONCLUSIONS}

The mobile networks require more capacity in the future as the mobile data traffic is expected to grow significantly. The operators' strategies depend on the revenue and cost factors but also on the market conditions like competition and marketing decisions. This paper has focused on modeling the cost factors and using mobile offloading as a complementary solution for macrocellular networks. The results show the conditions when mobile offloading is profitable, how much offloading will there be and where the offloading access points should be installed.

Proposition 1 characterizes the operator's optimal strategy based on the cost parameters and the future demand. In the long-term, the offloading will be beneficial if Eq. (8) holds, i.e., if complementing macro networks with offloading is more profitable than using only the macro improvements. On the other hand, for smaller future demands the comparison between offloading and macro strategies is given in Proposition 1B-C.

Cognitive radio may enhance both the offloading and macrocellular networks, which could be modeled as a change of 
parameters in the model. The cognitive radio technologies may help choosing the suitable frequency and access point based on the device's local radio environment. This way the quality of service can be improved and some of the capacity problems can be avoided. For example, if the macro network is capacitylimited then the device can switch to an offloading access point to relieve some of the load from the macro network.

\section{REFERENCES}

[1] Ericsson, "Traffic and Market Data Report," November 2011. [Online]. Available: http://www.ericsson.com/news/1561267

[2] Ficora, "Half-year report of communications market 2011. General market information." December 2011. [Online]. Available: http://www.ficora.fi/en/index/tutkimukset/generalmarketinformation.html

[3] Finnish Ministry of Transport and Communications, "Making broadband available to everyone. The national plan of action to improve the infrastructure of the information society," 2008, [publication; 50/2008].

[4] T. Smura, "Access alternatives to mobile services and content: analysis of handset-based smartphone usage data," in International Telecommunications Society 17th Biennial Conference, 2008, pp. 24 -27.

[5] M. Chowdhury and J. Noll, "Collaborative radio: Towards sustainable business in the evolution of 4g," in Wireless and Mobile Communications (ICWMC), 2010 6th International Conference on, sept. 2010, pp. 538 $-543$.

[6] Saunders, S., "Femtocell Evolution: A Model of Market and Standards Cooperation," July 2011. [Online]. Available: http://www.etsi.org/WebSite/homepage.aspx

[7] D. Calin, H. Claussen, and H. Uzunalioglu, "On femto deployment architectures and macrocell offloading benefits in joint macro-femto deployments," Communications Magazine, IEEE, vol. 48, no. 1, pp. 26 -32 , january 2010 .

[8] B. Han, P. Hui, V. Kumar, M. Marathe, J. Shao, and A. Srinivasan, "Mobile data offloading through opportunistic communications and social participation," Mobile Computing, IEEE Transactions on, vol. PP, no. 99 , p. $1,2011$.
[9] W. Lehr and J. Crowcroft, "Managing shared access to a spectrum commons," in IEEE International Symposium on Dynamic Spectrum Access Networks (DySPAN), 2005, pp. 420-444.

[10] I. Akyildiz, W. Lee, M. Vuran, and S. Mohanty, "Next generation/dynamic spectrum access/cognitive radio wireless networks: a survey," Computer Networks, vol. 50, no. 13, pp. 2127-2159, 2006.

[11] J. Peha, "Sharing spectrum through spectrum policy reform and cognitive radio," Proceedings of the IEEE, vol. 97, no. 4, pp. 708-719, 2009.

[12] A. Saatsakis, P. Demestichas, V. Merat, C. Le Page, T. Loewel, and K. Nolte, "Femtocell and flexible base station cognitive management," in Personal, Indoor and Mobile Radio Communications, 2009 IEEE 20th International Symposium on, sept. 2009, pp. $1-5$.

[13] Tardy, I. and Grondalen, O., "Which regulation for cognitive radio? An operator's perspective," January 2010. [Online]. Available: http://www.etsi.org/WebSite/homepage.aspx

[14] CEPT, "Technical and operational requirements for the possible operation of cognitive radio systems in the 'white spaces' of the frequency band 470-790 MHz," CEPT ECC Report 159, January 2011.

[15] FCC, "Unlicensed operation in the TV broadcast bands," Second Memorandum Opinion and Order, FCC 10-174, September 2010.

[16] K. Johansson, "Cost effective deployment strategies for heterogeneous wireless networks," Ph.D. dissertation, KTH Communication Systems, SE-100 44 Stockholm, Sweden, November 2007.

[17] J. Harno, "Techno-economic valuation of mobile communications scenarios," Ph.D. dissertation, Aalto University, P.O. Box 13000, FI-00076, Espoo, Finland, October 2010.

[18] FemtoForum, "Femtocells - Natural Solution for Offload," July 2010. [Online]. Available: http://femtoforum.org

[19] Signal Research Group, "Femto Forum femtocell business case whitepaper," April 2009. [Online]. Available: http://femtoforum.org 